\title{
A New Plausible Link between Lysophosphatidylcholine, TGF- $\beta$, and Fibromuscular Dysplasia
}

\author{
Michio Shimabukuro
}

Department of Cardio-Diabetes Medicine, Institute of Biomedical Sciences, Tokushima University Graduate School, Tokushima, Japan

\section{See article vol. 23: 673-680}

Fibromuscular dysplasia (FMD) is a relatively rare and non-atherosclerotic disease of medium-sized vessels that was originally described in $1938^{1)}$. It is characterized by arterial dysplasia and obstruction to arterial blood flow caused by neointimal lesions rich with cells that have smooth muscle phenotype. Patients with FMD can present with arterial stenosis, beading, dissection, and aneurysm. In the US Registry for FMD cohort $^{2,3)}$, nearly $80 \%$ of registrants had renal FMD (presenting as hypertension), and almost three-quarters had carotid FMD (presenting as stroke or transient ischemic attack). Vertebral FMD was the third most common condition (36.6\%). Other reported sites of involvement include the mesenteric arteries, iliac arteries, intracranial vessels, and upper extremity (brachial) arteries ${ }^{2,3)}$. Incidences of FMD are more common among females, accounting for approximately $90 \%$ of the adult cases. Although FMD was thought to be a disease in young women, recent studies revealed that older individuals account for a large proportion of the total affected patients ${ }^{2,3)}$. High prevalence of aneurysms (23.5\%), sudden death (19.8\%), and stroke $(53.5 \%)$ among first- and second-degree family members in the US Registry suggests that FMD may represent an inherited systemic arteriopathy with diverse clinical phenotype ${ }^{2,3)}$. However, the genetic and biological determinants of FMD have been largely unknown ${ }^{2)}$.

The recent report by Tanaka, et al. may provide an insight into the underlying mechanism(s) of $\mathrm{FMD}^{4)}$. The authors compared the distribution of lipid molecules, between atherosclerotic visceral artery

Address for correspondence: Michio Shimabukuro, Department of Cardio-Diabetes Medicine, Institute of Biomedical Sciences, Tokushima University Graduate School, 3-18-15

Kuramoto, Tokushima 770-8503, Japan

E-mail: mshimabukuro-ur@umin.ac.jp

Received: February 12, 2016

Accepted for publication: February 17, 2016 aneurysms (VAAs) and FMD-associated VAAs, using an emerging technique called matrix-assisted laser desorption/ionization imaging mass spectrometry (MALDI-IMS $)^{4,5)}$. This method can distinguish various types of lipid molecules, such as phospholipids, neutral lipids, glycolipids, and fatty acids, at the molecular species level in each arterial layer ${ }^{5)}$. The authors found a characteristic distribution of cholesterol esters in the intima and media of atherosclerotic VAAs, which was negligible in FMD-associated VAAs. By contrast, lysophosphatidylcholine (lysoPC) accumulated in the medial ridge of the adventitia in the aneurysmal sac and the adjacent arteries of patients with FMD-associated VAAs. LysoPC accumulated mainly in the area of intimal hyperplasia in atherosclerotic VAAs and the adjacent arteries.

Although overlapping features between FMD and vascular connective tissue diseases, such as LoeysDietz syndrome or the vascular type of Ehlers-Danlos syndrome, have been suggested, the prevalence of genetic mutations associated with connective tissue disease was negligible in a cohort of FMD patients ${ }^{2,6)}$. In the cohort, two FMD patients with a history of arterial dissection and aortic ectasia or aneurysm showed distinct novel point mutations in transforming growth factor (TGF)- $\beta$ receptor type 1 gene $^{6}$. Ganesh et al. found that plasma levels of circulating TGF- $\beta 1$ and TGF- $\beta 2$ and secretion of both factors by fibroblasts were increased in FMD patients than in the matched controls ${ }^{7}$. LysoPC, with one mole of fatty acid per mole of lipid in the $s n-1$ position, is produced by the hydrolysis of dietary and biliary phosphatidylcholine, but it is also formed in most tissues via hydrolysis of phosphatidylcholine by the phospholipase A2 enzyme superfamily as part of the de-acylation/re-acylation $\mathrm{cycle}^{8)}$. Though stearoyl-lysoPC shows an anti-inflammatory role, lysoPC has proinflammatory properties and is known to be a pathological component of oxidized lipoproteins (LDL) that is found in the plasma and atherosclerotic lesions. LysoPC is thought to be a mediator of TGF- $\beta$ activation $^{6}$. Although it is currently unclear whether the 
The Lands' cycle

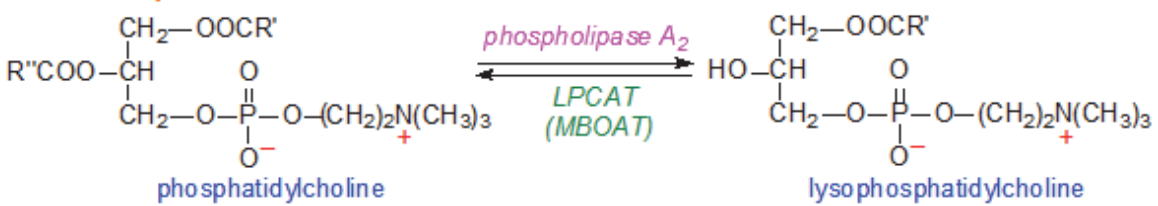

http://www.lipidhome.co.uk/lipids/complex/pc/index.htm.
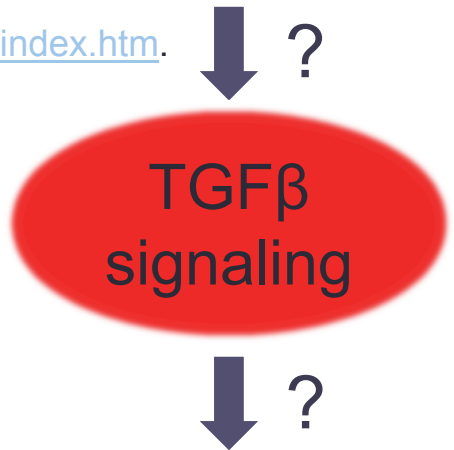

Fibromusclurar dysplasia (FMD)
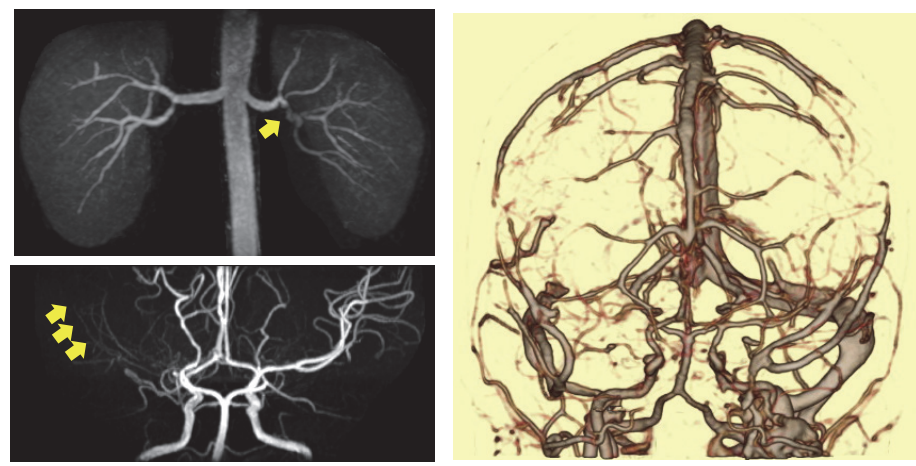

Fig. 1. Possible underlying mechanism in fibromusclurar dysplasia (FMD)

TGF $\beta$ : Transforming growth factor beta

accumulation of lysoPC is associated with the dysregulation of TGF- $\beta$ signaling, the potential involvement of TGF- $\beta$ pathways in the pathogenesis of FMD is an area for future investigation (Fig. 1), especially as this pathway could provide a potential target for diseasemodifying therapies.

There are two new questions to be elucidated. Firstly, FMD can be classified histologically (on the basis of the affected arterial layer) into intimal fibroplasia, medial dysplasia, or adventitial (periarterial) fibroplasia ${ }^{9}$. The current study did not distinguish between histological types, however, the involvement of lysoPC should be evaluated with respect to each histological class. Medial fibroplasia represents the most common dysplastic lesion, accounting for more than $80 \%$ of the fibromuscular lesions, and is angiographically characterized by the classic "string of beads" appearance due to alternating fibromuscular webs and aneurysmal dilatation. In areas of dilatation, the internal elastic lamina is absent, which is possibly the primary defect. Intimal fibroplasia, which accounts for $-10 \%$ of FMD, is caused by circumferential or eccentric deposition of collagen in the intima, with no inflammatory or lipid component. Medial hyperplasia, a rare manifestation, is caused by smooth muscle cell hyperplasia without fibrosis. Periarterial hyperplasia, also a rare manifestation, is caused by an expansion of the fibrous adventitia, such that collagen extends into the periarterial fat, accompanied by inflammation. Secondly, gene-environment interactions in FMD susceptibility ought to be considered. Two proposed interactions are smoking and estrogen ${ }^{3,10)}$. Savard et al. demonstrated that patients with renal FMD have a much higher smoking rate than the matched hypertensive controls ${ }^{10)}$. The remarkable predilection for female patients in the FMD population 
(>90\%) suggests that estrogen and other hormonal factors are major contributors in the development of FMD.

\section{Conflicts of Interest}

None.

\section{References}

1) Leadbetter $W$ and Burkland CE: Hypertension in unilateral renal disease. J Urol, 1938; 39: 1-626

2) O'Connor SC and Gornik HL: Recent developments in the understanding and management of fibromuscular dysplasia. J Am Heart Assoc, 2014; 3: e001259

3) Olin JW, Gornik HL, Bacharach JM, Biller J, Fine LJ, Gray BH, Gray WA, Gupta R, Hamburg NM, Katzen BT, Lookstein RA, Lumsden AB, Newburger JW, Rundek T, Sperati CJ and Stanley JC: Fibromuscular dysplasia: state of the science and critical unanswered questions: a scientific statement from the American Heart Association. Circulation, 2014; 129: 1048-1078

4) Tanaka H, Zaima N, Sasaki T, Yamamoto N, Inuzuka K, Sano M, Konno H, Urano T, Setou M and Unno N: Characteristic Distribution Pattern of Lysophosphatidylcholine in Fibromuscular Dysplasia-Associated Visceral Artery Aneurysms Compared with Atherosclerotic Vis- ceral Artery Aneurysms. J Atheroscler Thromb, 2016; 23: 673-680

5) Zaima N, Sasaki T, Tanaka H, Cheng XW, Onoue $K$, Hayasaka T, Goto-Inoue N, Enomoto H, Unno N, Kuzuya $M$ and Setou M: Imaging mass spectrometrybased histopathologic examination of atherosclerotic lesions. Atherosclerosis, 2011; 217: 427-432

6) Poloskey SL, Kim E, Sanghani R, Al-Quthami AH, Arscott P, Moran R, Rigelsky CM and Gornik HL: Low yield of genetic testing for known vascular connective tissue disorders in patients with fibromuscular dysplasia. Vasc Med, 2012; 17: 371-378

7) Ganesh SK, Morissette R, Xu Z, Schoenhoff F, Griswold BF, Yang J, Tong L, Yang ML, Hunker K, Sloper L, Kuo S, Raza R, Milewicz DM, Francomano CA, Dietz HC, Van Eyk J and McDonnell NB: Clinical and biochemical profiles suggest fibromuscular dysplasia is a systemic disease with altered TGF-beta expression and connective tissue features. Faseb j, 2014; 28: 3313-3324

8) http://www.lipidhome.co.uk/lipids/complex/pc/index.htm.

9) Olin JW, Bakris GL and Forman JP: Clinical manifestations and diagnosis of fibromuscular dysplasia. In: Post TW (Ed), UpToDate, Waltham, MA. (Accessed on February 12,2016$)$

10) Savard S, Azarine A, Jeunemaitre X, Azizi M, Plouin PF and Steichen O: Association of smoking with phenotype at diagnosis and vascular interventions in patients with renal artery fibromuscular dysplasia. Hypertension, 2013; 61: $1227-1232$ 